\title{
ISO 14001 y variables económicas, ¿hay alguna relación? Análisis de las empresas certificadas del sector cerámico español
}

\author{
A. PEIRÓ-SIGNES', M. SEGARRA-OÑA', J. MONDÉJAR- JIMÉNEZ², M. VARGAS-VARGAS² \\ 'Departamento de Organización de Empresas, Universitat Politècnica de València. \\ ${ }^{2}$ Departamento de Estadística. Universidad de Castilla La Mancha
}

\begin{abstract}
Es un hecho constatable el incremento de la adopción de prácticas medioambientales certificadas de manera formal a través de sistemas de gestión medioambiental. De su implantación, que tiene un elevado coste para las empresas, se espera la generación de beneficios, aunque no se ha documentado aún si existe relación entre la implantación de la ISO 14001 y su influencia en la mejora de los indicadores económicos a lo largo del tiempo. En este trabajo se analiza la relación entre la implantación de un sistema de gestión medioambiental, la ISO 14001, y el comportamiento económico en el corto, medio y largo plazo de las empresas del sector cerámico español. Se estudian los indicadores económicos para cada una de las 66 empresas productoras que han implantado la citada norma desde 1996 hasta el año 2009 a través de la comparación con su grupo de control. Los resultados indican que la ISO 14001 no influye en los resultados de las empresas del sector cerámico, ya que no se han encontrado diferencias significativas en los valores de ingresos de explotación ni en el incremento de los ingresos a largo plazo en ninguno de los tres horizontes temporales, corto, medio y largo plazo, obteniendo resultados opuestos a los esperados.
\end{abstract}

Palabras clave: Sector cerámico español, ISO 14001, resultados económicos, SGM, event study

Analysis of the impact of ISO 14001 in the economic variables of the Spanish ceramic tile industry's companies.

The increased in the adoption of formally environmental certified practices through environmental management systems is a provable fact. Its implementation, which has a high cost for the companies, is expected to generate benefits, although the relation between the implementation of ISO 14001 and its influence on the improvement of economic indicators over time has not been documented. This paper analyzes the relation between the implementation of an environmental management system, the ISO 14001, and economic performance in the short, medium and long term for the companies of the Spanish ceramic tile industry. It explores the economic indicators for each of the 66 manufacturing companies which have implemented the aforementioned standard from 1996 until 2009 through a comparison with a control group. Results show that ISO 14001 does not affect the economic results of the studied companies. We have not found significant differences in the operating income values, neither in the increase in revenues in the long term in any of the three analyzed periods, contrary to the expected results.

Keywords: Spanish ceramic tile industry, ISO 14001, economic performance, EMS, event study.

\section{INTRODUCCIÓN.}

En un mundo donde las preocupaciones ambientales aumentan (1) y las barreras comerciales disminuyen, el consenso sobre la gestión ambiental y los estándares de certificación tiene gran atractivo para los sectores que operan a nivel internacional (2).

La gestión medioambiental es, en estos momentos, una cuestión clave para las empresas $(3,4)$. Por una parte, los cambios que la sociedad demanda $y$, por otra, la regulación medioambiental, llevan a un importante número de empresas a considerar opciones "verdes" como fuente de cambio estratégico y oportunidades competitivas (5), integrando la pro-actividad medioambiental en su estrategia general $(6,7)$.

Teniendo esto en consideración, se ha observado en los últimos años un importante aumento de la implantación de sistemas de control y de gestión que aseguran que los productos y servicios cumplen con los estándares de excelencia relativos a las prácticas sostenibles y medioambientalmente responsables (8) siendo uno de los más utilizados la norma ISO 14001 (9-11). Pero, además de generar beneficios debidos al control de los procesos y a la generación de información interna específica (12), son ya muchos los autores que apuntan a la mejora no sólo de indicadores operacionales, sino a la influencia que los sistemas de gestión medioambiental tiene sobre el control de costes y la mejora de la imagen de la compañía (13-16), lo que se traduce en un incremento de los resultados económicos (17). La implantación de la norma ISO 14000 y cómo afecta al comportamiento empresarial se ha estudiado en diversos sectores a nivel internacional como el metalmecánico (18), 
el hotelero (19), el sector de la construcción (20), la industria canadiense de papel y pulpa (21) o en complejos deportivos (22), aunque los estudios aplicados al sector cerámico aún son escasos $(11,23)$.

Así, siguiendo a Espí (24), la profundización en el estudio de la sostenibilidad de la industria cerámica así como de la creación de valor a través de la certificación medioambiental como indican, entre otros, Sheppard et al. (23) y, en particular estudiando las implicaciones que puede tener la implantación de la ISO 14001, en la línea de trabajo de Heras - Saizarbitoria et al. $(25,26)$ es totalmente pertinente en un contexto concreto de crisis económica y en un sector específico, el de fabricación de baldosas cerámicas, que está orientado hacia el sector exterior en el que la armonización de criterios medioambientales es cada vez más importante.

La Norma ISO 14001 está siendo implantada por un número cada vez mayor de empresas $\mathrm{y}$, aunque algunos autores han estudiado aspectos relacionados con la adopción de prácticas medioambientales certificadas de manera formal a través de sistemas de gestión medioambiental y su impacto sobre las decisiones en la cadena de valor a nivel genérico (28-30), poco se ha estudiado hasta el momento sobre cómo impacta la implantación de la norma ISO14001 en los resultados empresariales en el sector cerámico español a lo largo del tiempo.

Teniendo en cuenta lo expuesto anteriormente, el objetivo de este trabajo consistirá en evaluar el impacto que la implantación de una herramienta de gestión medioambiental, la norma ISO 14001, tiene en las empresas del sector cerámico español sobre los resultados económicos de las mismas a corto, medio y largo plazo a través de técnicas estadísticas avanzadas.

En este trabajo se revisa la literatura existente sobre implantación de la norma ISO14001 y su aplicación al sector cerámico para posteriormente analizar y evaluar la muestra total de 66 empresas del sector que han implantado la norma desde 1996 hasta 2009 con respecto a su grupo de comparación. El artículo documenta el efecto de la inversión en la implantación de la ISO 14001 sobre los principales indicadores económicos empresariales ${ }^{1}$ y se establecen relaciones empíricas entre la gestión medioambiental a través de la herramienta estudiada y el desempeño empresarial. Tras el análisis estadístico de los resultados y su discusión, se finaliza el trabajo resumiendo las conclusiones del mismo.

\section{REVISIÓN DEL ESTADO DEL ARTE Y PLANTEAMIEN- TO DE HIPÓTESIS.}

Son numerosos los trabajos que analizan el impacto económico de las empresas que implantan un sistema ISO 14001, encontrando evidencias empíricas para afirmar que la ISO 14001 mejora tanto los resultados medioambientales como los económicos a través de la reducción de costes debido a una generación menor de residuos, del ahorro de energía, del cumplimiento de legislación de la mejora de la calidad o de la mejora de su reputación $(14,19,25,26,30)$.

Estas mejoras se justifican en que la ISO 14001 contribuye a generar unos recursos tangibles e intangibles valiosos para

${ }^{1}$ Se analizan: Ingresos de explotación (mil EUR), EBIT (mil EUR), EBITDA (mil EUR) Rentabilidad económica o ROA (\%), Margen de beneficios (\%),Beneficio por empleado (mil EUR), Coste medio por empleado al año (mil EUR). mantener o crear ventajas competitivas (8). Otros autores (30, 31) sostienen que los sistemas de gestión ambiental aportan a la empresa un sistema de información que no sólo reduce la contaminación sino que también contribuye a la mejora del resultado corporativo.

Heras-Saizarbitoria et al., $(25,26)$ han estudiado en detalle la implantación de los sistemas de gestión medioambiental en las empresas, haciendo especial hincapié en el estudio de las motivaciones que conduce a las empresas a adoptar la norma ISO 14001 (25) así como en estudiar la relación entre la certificación medioambiental y el resultado financiero (26). Nuestro trabajo presenta varias diferencias que conviene remarcar y que permiten diferenciar significativamente un estudio de otro. En este análisis se ha tomado el año de la certificación como referencia para valorar el comportamiento económico a partir del suceso, mientras en los trabajos anteriores de Heras-Saizarbitoria et al. (26) se dividen las empresas entre certificadas, todavía no certificadas y no certificadas, por lo que la influencia del evento en los resultados es menos clara. También se han acotado en nuestro trabajo las empresas que forman parte del grupo de comparación, de forma que se garantiza que éstas tienen un resultado similar en el año del evento y, por tanto, valoramos la evolución a partir del evento de empresas que se encuentran en una situación de partida similar y se establecen grupos de comparación homogéneos para determinar las diferencias entre las empresas certificadas y no certificadas en lugar de realizar una comparación conjunta de los resultados de todas las empresas de cada grupo.

Por otra parte, el estudio abarca un periodo de 10 años, incluyendo parte de la crisis económica, lo que permite valorar si la ISO 14001 está sirviendo para mejorar la posición competitiva de las empresas. Incluye empresas en todos los estadios de desarrollo, empresas en las que la norma está recién certificada y otras en las que el tiempo de implantación es mayor.

Los fabricantes de productos cerámicos están prestando una cada vez mayor atención a la protección del medio ambiente $(33,34)$, en la medida permitida por logros tecnológicos así como por la optimización de sus capacidades innovadoras y opciones estratégicas en la línea de trabajo apuntada por diversos trabajos previos (35-40).

La mejor organización de la producción para la optimización de los recursos naturales a fin de satisfacer sus necesidades actuales y futuras integrando los objetivos de sostenibilidad se ha convertido en un objetivo prioritario para la industria cerámica a nivel global $(31,41)$.

La Norma ISO 14001 es el estándar de la gestión ambiental más utilizado a nivel mundial (42) con una cada vez mayor implantación en el sector cerámico español, como se puede observar en la figura 1.

Hasta el momento, aunque la cantidad de estudios relativos a los motivos de su implantación o de los beneficios de imagen y comerciales, es considerable $(42,43)$, no se ha estudiado aún la relación entre la implantación de la norma ISO 14001 y los resultados económicos de las empresas dependiendo del año de implantación, ni se ha hecho este estudio en el sector cerámico, en el que crecen las certificaciones de manera importante año tras año y que parece estar firmemente decidido a tener una actitud cada más proactiva a nivel medioambiental $(24,33,34,40)$, ni se ha estudiado cuándo empieza a afectar a la empresa la implantación de la misma. 
De todo lo expuesto, se desprende que los beneficios derivados de la implantación de un sistema de gestión medioambiental no se observan de manera inmediata, pero sí se espera que las empresas que han adoptado esta ecoinnovación organizativa, tengan beneficios tangibles a medio y largo plazo. El corto plazo en este trabajo se ha considerado de un año, el medio plazo más de un año y menos de cuatro y largo plazo, más de cuatro años. Para la definición de corto, medio y largo plazo se han seguido criterios económicos, por el que corto plazo se considera aquel perído durante el cual los factores de producción fijos no pueden cambiarse, pero puede alterarse el nivel de utilización de los factores y que se considera habitualmente inferior a los doce meses, el medio plazo en términos económicos hace referencia a cualquier período de tiempo comprendido entre el largo y el corto plazo, el largo plazo, según el Plan General de Contabilidad, se considera el tiempo del proceso de producción durante el cual es posible variar todos los factores productivos, se considera a más de cinco años aunque depende del entorno y para el caso del sector cerámico y ante la situación cambiante actual, se considera de cuatro años para realizar el análisis.

Basándonos en lo anteriormente expuesto, planteamos nuestras hipótesis de trabajo:

H1: La implantación de la norma ISO 14001 en las empresas del sector cerámico español no afecta al resultado económico a corto plazo.

H2: La implantación de la norma ISO 14001 en las empresas del sector cerámico español genera incrementos del resultado económico a medio plazo.

H3: La implantación de la norma ISO 14001 en las empresas del sector cerámico español genera incrementos del resultado económico a largo plazo.

\section{METODOLOGÍA.}

\subsection{Muestra y recolección de la información.}

El primer paso a la hora de realizar el estudio consiste en la determinación de las empresas pertenecientes al sector cerámico según su clasificación CNAE 2009 y certificadas mediante la ISO 14001. Para ello se ha utilizado

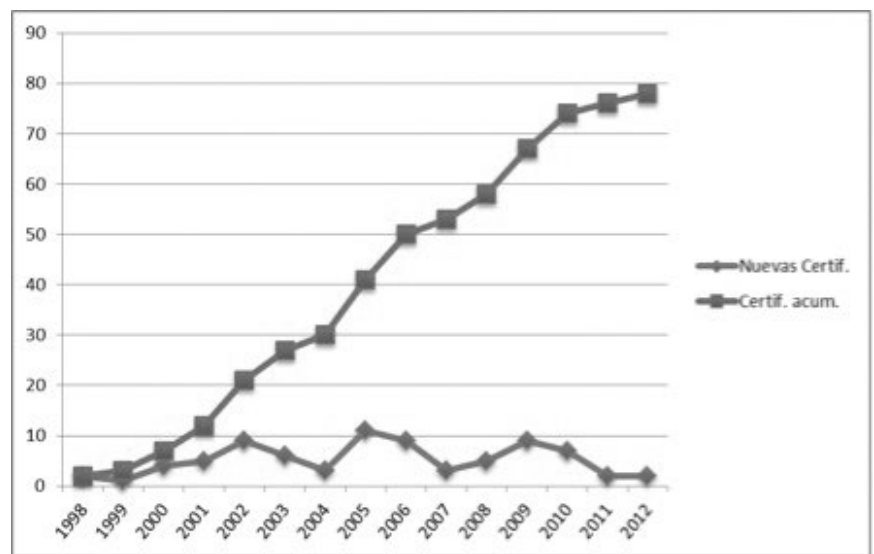

Figura 1.- Evolución del número de empresas certificadas según la norma ISO14001 en el sector cerámico español desde 1998 hasta 2012. principalmente la base de datos $\mathrm{IHOBE}^{2}$, así como las bases de datos disponibles en las páginas web de distintas entidades de certificación (TUV Reihland y SGS) y los listados de empresas certificadas proporcionados a los autores por las entidades de certificación (AENOR, DNV y APPLUS). Estos datos han sido completados a través de las páginas web de las empresas certificadas cuando ha sido necesario (año de certificación, empresa certificadora, etc.).

Como resultado de este trabajo de preparación de datos se identificaron 80 empresas certificadas hasta enero de 2012. De las empresas certificadas se descartaron un total de 13; 11 de ellas por estar certificadas entre 2010 y 2012 ni siendo posible evaluar su repercusión en el corto plazo por la ausencia de datos económicos a partir de 2010 y, 2 por falta de información económica en la base de datos $\mathrm{SABI}^{3}$.

Por otra parte, dentro del grupo de empresas certificadas de la muestra, en 2 casos no se pudo obtener datos económicos en el año de certificación por estar estas certificadas antes de 1999. En estos casos se optó por coger como año base de comparación el primer año con cuentas disponibles, 1999 y 2000, respectivamente, tras comprobar que su inclusión no afectaba a los resultados del estudio.

Las empresas descartadas no se incluyeron tampoco dentro de los grupos de comparación. Los grupos de comparación no deben incluir empresas certificadas e idealmente no deberían incluir ninguna empresa que hubiera introducido un sistema de gestión medioambiental. Para minimizar el posible impacto que esto podría tener sobre el estudio, se comprobó que ninguna de las empresas de los grupos de comparación hubiera obtenido la certificación EMAS o LEEDs, las dos certificaciones medioambientales más comunes después de la ISO. Por otra parte, hay que considerar que pocas empresas inician la implantación de sistemas de gestión medioambiental sin tener como uno de sus objetivos la obtención de la certificación y que, de media, 40 empresas forman parte de cada grupo de comparación, por lo que, en su caso, la inclusión de alguna empresa con las características indicadas en un grupo tendría una repercusión mínima sobre los indicadores utilizados en este estudio y, consecuentemente, sobre los resultados.

Por tanto, entendemos que las probabilidades de que los resultados puedan estar afectados por la inclusión de alguna empresa que disponga de un Sistema de Gestión Medioambiental implantado y no certificado son muy bajas.

De esta forma, el estudio se realizó sobre un total de 66 empresas certificadas. Los datos económicos en todos los casos se han obtenido de la base de datos SABI (Sistema de Análisis de Balances Ibéricos), que recoge información de las principales variables económicas por sector, descripción de la actividad por códigos CNAE, número de empleados, cifras de ventas de los últimos años, localización y ratios relacionados tanto con la productividad, como con el comportamiento económico y financiero.

Para estimar los efectos económicos que la implantación de la norma ISO 14001 tiene sobre las empresas se han considerado las variables económicas que se presentan en

\footnotetext{
${ }^{2}$ IHOBE, , http: / / www.ihobe.net

${ }^{3}$ SABI, (Sistema de Análisis de Balances Ibéricos), base de datos que recoge información de las principales variables económicas por sector, descripción de la actividad por códigos CNAE, número de empleados, cifras de ventas de los últimos años, localización y ratios relacionados tanto con la productividad, como con el comportamiento económico y financiero.
} 
la tabla I y que se han utilizado previamente para el análisis del comportamiento económico en trabajos previos $(19,44$, $45,46)$, lo que justifica su uso y su validez.

Para el estudio, se ha seleccionado un grupo de comparación para cada una de las empresas certificadas con el objeto de reducir y controlar aquellos efectos no relacionados con la implantación de la ISO 14001 que pueden afectar al comportamiento económico.

\subsection{Metodología para la estimación de efectos en el corto,} medio y largo plazo.

Siguiendo el procedimiento establecido por Hendricks et al. (47) para valorar el impacto en los resultados de las empresas de la implantación de sistemas de planificación de la producción, hemos creado para el estudio la variable "Indicador de Resultados Anormales, IRA". Estimamos los resultados anormales como el cambio en los resultados de la empresa de la muestra con respecto al cambio en la media de los resultados del grupo de comparación. Formalmente,

$$
\operatorname{IRA}=\left(N_{\mathrm{i}}\left(\mathrm{t}_{2}\right)-\mathrm{NR}_{\mathrm{i}}\left(\mathrm{t}_{1}\right)\right)-\left(\mathrm{NRG}_{\mathrm{i}}\left(\mathrm{t}_{2}\right)-\mathrm{NRG}_{\mathrm{i}}\left(\mathrm{t}_{1}\right)\right)
$$

Donde

$N R i(t 1)$ es el nivel de resultado de la empresa $i$ en el periodo $t 1$

$N R i(t 2)$ es el nivel de resultado de la empresa $i$ en el periodo $t 2$

NRGi(t1) es la mediana de resultado del grupo de comparación de la empresa $i$ en el periodo $t 1$

NRGi(t2) es la mediana de resultado del grupo de comparación de la empresa $i$ en el periodo $t 2$
El IRA se puede considerar como el cambio en la variable o como el cambio porcentual en la variable. Por ejemplo, una empresa puede tener un incremento de la rentabilidad financiera del $2 \%$ al $3 \%$, lo que supone un cambio en su rentabilidad financiera del $1 \%$ o un porcentaje de incremento de su rentabilidad financiera de un $50 \%$. Puesto que el nivel de rentabilidad financiera puede tener valores negativos, el uso de porcentajes de cambio no tiene sentido y llevaría a tener que prescindir de los datos negativos, lo que resultaría en una pérdida de potencia estadística y en una alteración de los datos que pondría en cuestión los resultados.

Por tanto, se ha calculado en todos los casos el cambio de la variable en lugar del cambio porcentual de la variable. De esta forma, para valorar el indicador de resultados anormales para la variable ROA entre el año t2 y t1 la fórmula quedaría:

$$
\operatorname{IRA}_{\mathrm{ROA}}=\left(\operatorname{ROA}_{\mathrm{i}}\left(\mathrm{t}_{2}\right)-\operatorname{ROA}_{\mathrm{i}}\left(\mathrm{t}_{1}\right)\right)-\left(\operatorname{ROA}_{\mathrm{Gi}}\left(\mathrm{t}_{2}\right)-\operatorname{ROA}_{\mathrm{Gi}}\left(\mathrm{t}_{1}\right)\right)
$$

Siendo $\mathrm{ROA}_{\mathrm{Gi}}$ la mediana del grupo de comparación para el periodo especificado. De esta forma, se calculan los indicadores de resultado anormal para cada una de las variables de estudio.

Por otra parte, la inclusión de variables absolutas, como Importe de Ventas, Beneficios antes de Impuestos, $\mathrm{u}$ otras en el cálculo de los indicadores llevaría a cometer errores de apreciación al estar determinados los grupos de comparación en base a un ratio (Rentabilidad Económica). Por ejemplo, una empresa de la muestra podría tener un importe de ventas mucho mayor que la media de su grupo de comparación, suponiendo que tanto la empresa de

\begin{tabular}{|c|c|c|c|c|}
\hline & Mínimo & Máximo & Media & Desv. típ. \\
\hline Ingresos de explotación(mil EUR) & 135,81 & 431901,00 & 29532,08 & 62605,96 \\
\hline Resultado de explotación (mil EUR) & $-5537,24$ & 29100,21 & 1255,08 & 6187,84 \\
\hline Result. ordinarios antes Impuestos (mil EUR) & $-5806,39$ & 50050,00 & 1236,04 & 7825,31 \\
\hline $\begin{array}{l}\text { Rendimiento del capital invertido } \\
\text { (Return on Capital Employed, } \% \text { ) }\end{array}$ & $-30,93$ & 28,35 & $-0,04$ & 8,33 \\
\hline Rentabilidad económica o ROA $(\%)$ & $-24,69$ & 27,43 & $-1,45$ & 6,77 \\
\hline Margen de beneficios (\%) & $-70,35$ & 147,85 & $-3,38$ & 25,90 \\
\hline Rotación de activos & 0,03 & 4,93 & 0,83 & 0,82 \\
\hline Beneficio por empleado (mil EUR) & $-59,84$ & 284,03 & $-0,45$ & 44,35 \\
\hline Beneficio operacional por empleado (mil EUR) & 27,16 & 592,67 & 196,80 & 138,75 \\
\hline (Coste /Beneficios operativos) por empleado (\%) & 5,77 & 87,64 & 26,37 & 17,81 \\
\hline Coste medio por empleado al año (mil EUR & 16,77 & 56,40 & 35,36 & 9,21 \\
\hline Activos totales por empleado (mil EUR) & 37,01 & 1764,76 & 465,05 & 340,32 \\
\hline Número empleados Ultimo año & 5,00 & 2084,00 & 157,65 & 287,27 \\
\hline
\end{tabular}

TABLA I. VARIABLES ECONÓMICAS ANALIZADAS Y PRINCIPALES VALORES. 
la muestra como su grupo de comparación evolucionan constantemente incrementando sus ventas en la misma proporción, la empresa de la muestra reportaría siempre unas ventas superiores a las de su grupo de comparación y, esto quedaría reflejado en el indicador de comportamiento anormal positivo que no refleja una evolución de las ventas igual (proporcional a su tamaño).

Por esta razón, las variables que se pueden ver afectadas se presentan en función del incremento porcentual de la variable con respecto al año anterior (p. e. $\Delta \%$ Importe de las ventas). Siguiendo el mismo criterio, otros indicadores se presentan en función del número de empleados, lo que permite evaluar la eficiencia económica de las empresas certificadas respecto a su correspondiente grupo de comparación.

Para seleccionar las empresas que forman parte del grupo de comparación hay distintas alternativas, tanto en la forma de identificarlas como en la determinación del número que lo componen. Para la identificación de los grupos de comparación, los primeros estudios de eventos (event studies) que valoraban el resultado operativo se basaban en el análisis del sector y/o el tamaño (48-52). Por otra parte, entre los autores citados, algunos han utilizado una única empresa como elemento de comparación, mientras otros han utilizado un grupo de empresas. En este trabajo se ha seguido la guía propuesta por Barber y Lyon (53) para la selección de los grupos de comparación. Así, en primer lugar, utilizaremos un grupo de comparación frente a la utilización de una única empresa que haya tenido un resultado similar previo al evento valorado. Se aplica una metodología de 4 pasos basada en la selección de empresas atendiendo por una parte, al código SIC, equivalente al código CNAE español y, por otra, al valor del indicador de rentabilidad económica (en inglés ROA). De forma que:

- Paso 1. Para cada empresa de la muestra se identifican todas las empresas que coinciden con los dos primeros dígitos del código CNAE y cuyo valor de rentabilidad económica (o ROA) en el año de inicio de la medición se encuentra entre el 90 y el $110 \%$ del correspondiente a la empresa de la muestra. Todas las empresas que forman parte cumplan estos criterios pasan a formar parte del grupo de comparación de la empresa de la muestra.

- Paso 2. Si no se encuentran empresas en el paso 1, se amplía la búsqueda a las empresas con el mismo primer dígito del código CNAE y cuyo ROA se encuentra entre el 90 y el $110 \%$.

- Paso3.Si no encontramos empresas en el paso 2 ampliamos a búsqueda a cualquier empresa, independientemente del código CNAE, que cumpla con el filtro $90-110 \%$.

- Paso 4. Si no se encuentra ninguna empresa que cumpla lo anterior, se elige la empresa que tenga un resultado más cercano independientemente del código CNAE.

Para este estudio, hemos utilizado una variación sobre esta metodología que creemos que mejora el resultado y permite un análisis más potente al incidir sobre algunos de los inconvenientes que resultan de la aplicación de esta metodología.
En primer lugar, puesto que nuestra intención es estudiar el impacto de la implantación de la ISO 14001 en los resultados empresariales del sector cerámico, la búsqueda inicial dentro de los 2 primeros dígitos del código CNAE 2009 (equivalente al SIC en el estudio) nos llevaría a incluir todas las empresas con datos disponibles que se encuentran dentro del código 23 "Fabricación de otros productos minerales no metálicos", total 8869 empresas activas. La consideración de todas estas empresas presenta dos inconvenientes, primero, el control de las empresas certificadas, como se ha comentado anteriormente, y segundo, la inclusión de empresas de fabricación de vidrio, de tratamiento de piedra, de fabricación de productos abrasivos, que se alejan del objetivo de nuestra investigación.

Por tanto, para esta investigación consideramos más adecuado iniciar la búsqueda de empresas de los grupos de comparación dentro de los 3 primeros dígitos del código CNAE, de forma que utilizaremos los grupos 232 Fabricación de productos cerámicos refractarios, 233 - Fabricación de productos cerámicos para la construcción y 234 - Fabricación de otros productos cerámicos para la selección de las empresas. Con esta selección, disponemos de 1643 empresas para formar los grupos de comparación que han resultado suficientes para garantizar un adecuado tamaño de los mismos evitando así la incorporación de empresas de sectores diferentes que compiten en mercados muy distintos y cuya evolución en la última década no tiene porqué ser similar a la del sector estudiado.

Para extraer las observaciones a lo largo del tiempo para cada una de las empresas de la muestra tomamos como año base (año 0) el año en el que se produjo la primera certificación de cada una de las empresas. De esta forma, una empresa que obtuvo su primera certificación medioambiental ISO 14001 en el año 2005 tiene como año base el año 2005 ( $\mathrm{t} 0=2005)$, el año siguiente sería el año 1 (t1=2006) y así sucesivamente.

Por lo tanto, para cada una de las empresas de la muestra se determina el año base, se obtiene el valor de rentabilidad económica para dicha empresa en el año base y se calcula el rango $90-110 \%$ en torno a dicho valor. Posteriormente, se incluye dentro del grupo de comparación de la empresa de la muestra todas las empresas preseleccionadas en base a su código CNAE cuya rentabilidad económica en el año base estudiado se encuentre dentro del rango antes calculado.

Mediante este procedimiento se seleccionaron las empresas que para cada uno de los grupos de comparación de forma que la media de empresas en los grupos de comparación resultó ser de 39,42 para las 66 empresas de la muestra.

Una vez determinado el grupo de comparación, el cálculo de los valores de $I R A$ para cada variable se realiza a partir del año base (año de certificación), de forma que una empresa certificada en el año 2000 podrá tener hasta 10 valores de IRA (si no hay valores perdidos) puesto que se dispone de datos hasta el 2010. En cambio, una empresa certificada en el 2009 sólo dispondrá de 1 valor de IRA para cada variable, puesto que los valores anteriores a la certificación no se consideran en el estudio.

Para valorar los efectos a corto, medio y largo plazo que la implantación de la ISO 14001 tiene sobre cada una de las variables estudiadas, se agrupan los valores de IRA 


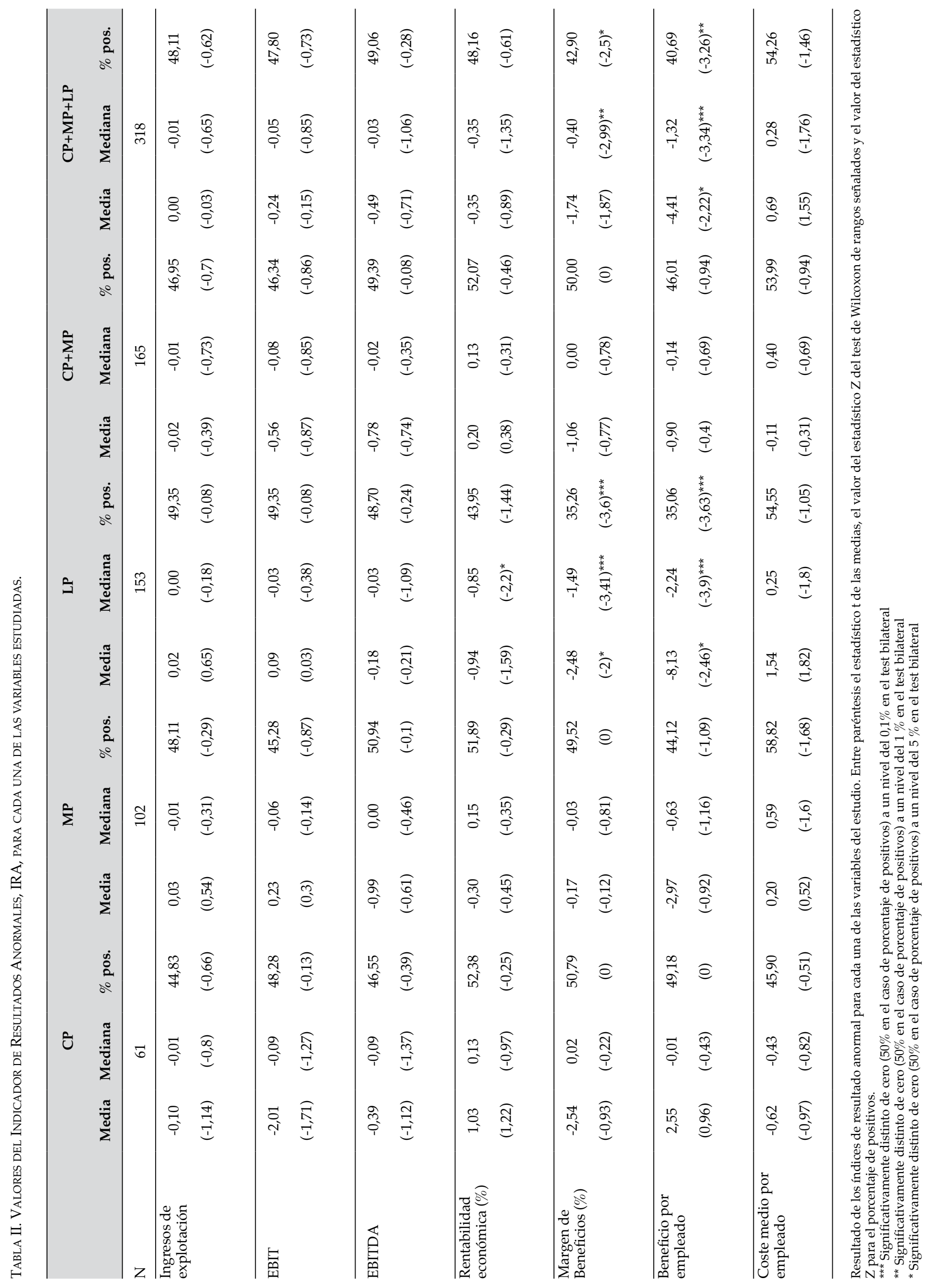


del primer año (corto plazo), del segundo y tercer año (medio plazo) y los valores a partir del 4 año (largo plazo), respectivamente. De esta forma, obtenemos una serie de datos que nos van a permitir el estudio estadístico de cada una de las variables en el corto, medio y largo plazo. Adicionalmente, hemos calculado el efecto global del corto y medio plazo y del corto, medio y largo plazo para valorar mejor los resultados obtenidos.

\section{DISCUSIÓN DE RESULTADOS.}

Después de describir el proceso de selección de la muestra y obtención de los valores del indicador IRA para cada una de las variables y para cada uno de los periodos temporales de estudio, y antes de realizar el análisis estadístico, conviene detectar y tratar los valores atípicos.

El control de los valores atípicos puede influir de forma importante en los valores de la media por lo que se estima oportuno eliminar de la muestras los valores de API calculados a partir de las medias en el nivel de 2,5\% de cada una de las colas de la distribución. A pesar de la eliminación de estos valores, los valores atípicos pueden estar influyendo todavía de forma importante en el resultado, por lo que se complementa el análisis paramétrico con el análisis mediante pruebas no paramétricas de la mediana y del porcentaje de empresas con resultados anormales positivos.

Para el estudio hemos utilizado el test $t$ de las medias sobre los IRAs de las variables seleccionadas. Además, se han realizado sobre los IRAs el test de Wilcoxon de rangos señalados (54), de forma que valoramos si la mediana de los IRAs es significativamente distinta de cero. Dentro de las pruebas no paramétricas, se incluye también el test binomial de signos para determinar si el porcentaje de firmas de la muestra que han experimentado un $I R A$ positivo es significativamente distinto del $50 \%$.

En la tabla II se muestran los resultados para cada una de las variables estudiadas y para cada uno de los periodos temporales establecidos del test $t$ de medias, test de Wilcoxon de rangos señalados de las medianas y test binomial de signos $^{4}$.

El test $t$ nos indica si la media de los IRAs de cada una de las variables es significativamente distinta de cero. Si la media de un correspondiente IRA es positivo, nos indica que el valor de la variable a la que representa el indicador es superior en la empresa de la muestra sobre la mediana de las empresas del grupo de comparación y viceversa. De la misma forma el test de Wilcoxon nos indica si la mediana de un correspondiente IRA es significativamente distinta de cero. Por otro lado, la prueba de signos nos indica el porcentaje de empresas que tienen un resultado significativamente distinto de cero, es decir el porcentaje de empresas de la muestra que tienen valores de la variable representada por el IRA superiores a los de su grupo de comparación. Por ejemplo, en el largo plazo tenemos que las empresas certificadas tienen de media un beneficio por empleado en el largo plazo significativamente inferior $(-8,13$ euros, $p<0,05)$ al de su grupo de comparación. Si consideramos el test de Wilcoxon diríamos que el Beneficio por empleado en el largo plazo para las empresas certificadas es 2,24 euros inferior $(p<0,001)$

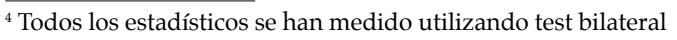

a la mediana de su grupo de comparación. Finalmente, según el test signos sólo el $35,06 \%$ de las empresas certificadas muestran un valor de beneficios por empleado superior al de la mediana de su grupo de comparación. Dicho de otra forma, las empresas certificadas muestran en un porcentaje significativo $(p<0,001)$ un valor de beneficios por empleado inferior al de su grupo de comparación. Por otra parte, si se observa estos mismos test para el medio plazo podemos decir que, de media, los beneficios por empleado de las empresas certificadas es 2,97 euros inferior a las de las empresas de su grupo de comparación aunque esta diferencia no es estadísticamente significativa.

Como se ha comentado anteriormente, el impacto de los valores atípicos (outliers) de IRA sobre la media puede ser importante a pesar de haber limitado los resultados extremos al 2,5\% en cada cola de la distribución. Es por ello por lo que se incide especialmente sobre los resultados obtenidos en las pruebas no paramétricas realizadas.

Los resultados en la primera columna de la tabla II permiten contrastar la hipótesis 1 que establecía que la implantación de la norma ISO 14001 en las empresas del sector cerámico español no afecta al resultado económico a corto plazo. Como se puede observar, ninguno de los indicadores económicos para los que realizan las pruebas resulta estadísticamente significativo, por lo que no podemos afirmar que existan diferencias entre los resultados económicos de las empresas certificadas con respecto a aquellas empresas pertenecientes a sus grupos de comparación.

Por otra parte, los resultados de las empresas certificadas en el medio plazo tampoco son estadísticamente diferentes de los que presentan las empresas de sus grupos de comparación, pues los IRAs de las variables estudiadas no son significativamente superiores a cero.

En cuanto a los resultados en el largo plazo, observamos que existen diferencias significativas en los indicadores de margen de beneficios y de beneficio por empleado. Al contrario de lo esperado, los valores de estos indicadores son mejores para las empresas pertenecientes a los grupos de comparación que para las empresas certificadas. El incremento del margen de beneficios en el largo plazo es significativamente inferior $(-1,49 \%, \mathrm{p}<0,001)$ en las empresas certificadas y sólo en el 35,06\% de los casos el margen de beneficios de las empresas certificadas se incrementó por encima del de los grupos de comparación.

El resto de indicadores presentan diferencias negativas aunque éstas no son estadísticamente significativas. Hay que destacar, en el caso de los costes por empleado, que un resultado mayor que cero indica que el incremento de los costes por empleado en el largo plazo superior en las empresas certificadas.

Finalmente, el análisis agregado para los periodos refuerza el resultado obtenido en el análisis individual de los distintos horizontes temporales. La diferencia de resultados agregados en el corto y medio plazo entre las empresas certificadas y no certificadas no son estadísticamente significativos. Sin embargo, teniendo en cuenta los resultados acumulados en el largo plazo, la mayoría de los indicadores de las empresas certificadas, excepto el porcentaje de beneficio y el beneficio por empleado ya indicados anteriormente, no muestra diferencias significativas respecto de las empresas no certificadas. 
En consecuencia, debemos rechazar las hipótesis 2 y 3 que establecían un mejor comportamiento económico de las empresas certificadas en el medio y largo plazo.

\section{CONCLUSIONES}

Esta investigación ha estudiado el efecto que una de las normas de gestión medioambiental de mayor implantación en estos momentos, la ISO 14001, tiene en los resultados económicos de las empresas del sector cerámico español. Se ha realizado un event study, analizando el comportamiento económico de las empresas que han implantado la citada norma, desde el año de su implantación y comparándolas con grupos de empresas que no tienen implantada la certificación y que son similares en cuanto a tipo de actividad y valor de rentabilidad económica.

Los resultados muestran, en primer lugar, que no existen diferencias significativas en el \% de incrementos de los ingresos de explotación en ninguno de los tres horizontes temporales, corto, medio y largo plazo. El incremento de los ingresos a largo plazo es de media un 3,9\% superior en las empresas certificadas y el porcentaje de empresas con mejores rendimientos sólo mejora un 3\% con la certificación, siendo en ambos casos no significativas desde el punto de vista estadístico.

Por otra parte, al analizar los ratios de rentabilidad, especialmente el ratio de rentabilidad económica que hace referencia al cociente entre el beneficio neto y el activo total y expresa la rentabilidad económica de la empresa, independientemente de la forma en que se financie el activo, se observa que el primer año tras la certificación el efecto es positivo, el $64 \%$ de las empresas certificadas lo hacen mejor que la media de su grupo de comparación y de media el incremento de la rentabilidad económica es de un punto porcentual, por lo que tendríamos una confirmación parcial de la primera de las hipótesis planteadas, que hace referencia a la mejora de los indicadores económicos en el corto plazo tras la implantación de la norma ISO 14001. Sin embargo, observamos que los resultados invierten la tendencia en el largo plazo con valores de resultados positivos peores que los correspondientes a los grupos de comparación.

Este resultado puede tener importantes implicaciones a nivel empresarial, ya que parece que una vez realizado el esfuerzo y la inversión y obtenida la certificación, se desaprovechan las oportunidades de mejora de resultados que la ISO 14001 como sistema de gestión medioambiental podría ofrecer a la empresa, como el incremento de información, reducción de costes o el control en el uso de los recursos energéticos, entre otros $(8,11,12,16,17,23,56)$.

Por último, en las variables representativas de la eficiencia de funcionamiento de la empresa, observamos que el coste medio por empleado empeora significativamente en el largo plazo. En el mismo sentido apuntan los resultados referentes a los beneficios por empleado a largo plazo, que son significativamente ( $p$-valor $<0,01$ ) menores en las empresas certificadas con respecto a las no certificadas de forma que el beneficio por empleado se reduce de media cerca de 8000 euros empleado y año. Este resultado parece el esperado teniendo en cuenta el comportamiento de otras variables relacionadas, incremento de los costes y activos por empleado y disminución del margen de beneficio.
En consecuencia, las hipótesis 2 y 3 deben ser rechazada, no podemos afirmar que la ISO 14001 influye positivamente en los resultados de las empresas del sector cerámico a medio y a largo plazo a tenor de los resultados, se debería profundizar en las razones por las cuales el comportamiento económico de las empresas seleccionadas en el largo plazo es opuesto al esperado.

Una de las razones que podría explicar este comportamiento es que el objetivo de las empresas que buscan la certificación medioambiental tiene su origen en un efecto estético y no de verdadera profundización y mejora del impacto medioambiental de la empresa (57), aspecto que debería hacer reflexionar a las empresas certificadas. Al realizar la inversión en la implantación de un SGM como la ISO 14001 los mayores beneficios se obtendrán de mejoras internas, al mejorar la eficiencia de sus procesos y actuaciones, lo que se refleja en mejores resultados económicos, y lograr una ventaja competitiva sostenible (58) ya que las mejoras derivadas de la imagen asociada a la sostenibilidad es una ventaja competitiva comparativa, fácilmente alcanzable por los competidores, que tendrá una duración limitada en el tiempo.

Esto último nos lleva a pensar si la forma de abordar las soluciones técnicas para la reducción del impacto ambiental está bien orientada y si las acciones complementarias informativas y formativas apoyan estas actuaciones, aspecto clave en el sector cerámico español como han estudiado previamente algunos autores $(59,60)$. Puede darse el caso de que una empresa realice una inversión importante en la instalación de un sistema de gestión medioambiental para controlar, procedimentar y reducir consumos sin haberse preocupado antes de concienciar a sus trabajadores en el uso de la misma.

Otra posible causa tendría que ver con el nivel de inversiones realizadas para obtener mejoras de rendimientos energéticos. Estas mejoras estarían lastrando los resultados económicos de las empresas al producirse una bajada importante de la producción y, consecuentemente del retorno de la inversión, pero este aspecto es una limitación de nuestro estudio, ya que no se ha considerado.

En este trabajo se ha puesto de manifiesto que, si bien en el corto plazo, la implantación de la norma ISO 14001 afecta positivamente a los resultados económicos de las empresas del sector cerámico, estos resultados no se mantienen a lo largo del tiempo, resultado llamativo e inesperado que debe hacer reflexionar a los empresarios.

Pese a las limitaciones que presenta el estudio, derivadas principalmente del momento de crisis económica de su aplicación lo que quizá ha afectado al comportamiento de los valores analizados y de la incapacidad de aislar totalmente los efectos, aunque la técnica utiliza controla las variables seleccionadas con respecto al resto $(47,48,53,54)$, lo que queda patente es el importante incremento de certificaciones según la norma ISO 14001 que se está produciendo en el sector y la importancia que para las empresas tiene conocer en detalle los beneficios (o la falta de ellos) que la implantación de la norma de estandarización de gestión medioambiental ISO 14001 puede tener.

Por otra parte, en el estudio se han utilizado únicamente técnicas de análisis cuantitativo, un estudio de tipo cualitativo, analizando en profundidad casos de implantaciones con éxito y fracasos, ayudaría a establecer 
los factores clave que diferencian una situación de la otra y a analizar qué pasa en esas situaciones concretas con los indicadores económicos.

Por último, parece interesante abrir una nueva vía de investigación recorriendo el camino inverso. Es decir, partiendo de la decisión de certificarse por parte de las empresas, sería conveniente determinar cómo se han comportado los indicadores económicos en los años previos a la certificación que puedan explicar el por qué unas empresas con unas determinadas características económicas decide certificarse y otra en la misma situación no.

\section{AGRADECIMIENTOS}

Los autores desean agradecer al Ministerio de Economía y Competitividad por el apoyo económico recibido a través del proyecto (EC02011-27369), así como a la Universitat Politècnica de València por la financiación a través de su programa de movilidad de profesores y del proyecto PAID06-2011-1879, "Impacto de las prácticas innovadoras en el "performance" medioambiental de la empresa: identificación de factores moderadores".

\section{REFERENCIAS}

1. M. Vargas-Vargas, M.L., Meseguer-Santamaría, J. Mondéjar-Jiménez, J.A. Mondéjar-Jiménez, Environmental Protection Expenditure for Companies: A Spanish Regional Analysis, Int. J. Environ. Res., 4, 373378, (2010).

2. G. Mullett, ISO 14000: Harmonizing Environmental Standards and Certification Procedures Worldwide, Minn. J. Global Trade (6): 379-400 (1997)

3. J.F. Molina-Azorín, E. Claver-Cortés, M.D. López-Gamero, J.J. Tarí, Green management and financial performance: a literature review, Manage Decis, 47 (7):1080-1100, (2009).

4. J. Rivas, M. Magadán, Less green taxes and more control over pollutant industries: a theoretical proposal, Environ. Eng. Manag. J., 9(9), 11731177, (2010).

5. M.E. Porter, C. Van der Linde, Toward a New Conception of the Environment-Competitiveness Relationship, J. Econ. Perspect., 9 (4), 97-118, (1995).

6. I. Martín-Tapia, J. A. Aragón-Correa, A. Rueda-Manzanares, Environmental strategy and exports in medium, small and microenterprises, Journal of World Business, 45 (3): 266-275, (2010).

7. J.C. Gázquez-Abad, J.F. Jiménez-Guerrero, J.A. Mondéjar-Jiménez, M. Cordente-Rodríguez, How Companies Integrate Environmental Issues Into Their Marketing Strategies, Environ. Eng. Manag. J., 10(12): 18091820, (2011)

8. G. Wever, Strategic environmental management: using TQEM and ISO 14000 Competitive Advantage, John Wiley and Sons, New York, NY, (1996).

9. M. Domico, J.M., Saidler, Clean, green manufacturing, Ceram Ind, 152 (2): 54-56, (2002).

10. V. Hocenski, Z. Hocenski, S.Vasilic, Application of Results of Ceramic Tiles Life Cycle Assessment due to Energy Savings and Environment Protection, Conference proceedings, 2972-2977.

11. R. Vasic, S. Despotovic, C. Lacnjevac, ISO 14001 Standard and its applications in ceramic industry, Br.Ceram.Proc., 60: 377-378 (1999).

12. N. Johnstone, J. Labonne J., Why do manufacturing facilities introduce environmental management systems? Improving and/or signalling performance? Ecol. Econ., 68: 719-730, (2009).

13. D. Duran, I. Duran, A. Diaconu, Issues on costs and investment for environmental protection, Environ. Eng. Manag. J., 8(4): 705-708, (2009).

14. B. Junquera, J. A. Del Brio, Environmental concurrent engineering: a way to competitive advantage?, World Review of Entrepreneurship, Management and Sustainable Development, 5, (3), 256-270, (2009).

15. G.J. Collins, S. M. Grimes, J. G. Boyce, Developing environmental performance indicators for an information technology systems and services company, Int. J. Environ. Pollut., 18 (3):260-270, (2002).

16. O. Boiral, J.F. Henri, Modelling the impact of ISO 14001 on environmental performance: A comparative approach, J. Environ. Manag., 99:84-97, (2012).
17. K.K. Cushing, H. McGray, H. Lu, Understanding ISO 14001 adoption and implementation in China, Int. J. Envir. Sust. Develop., 4(3):246-268, (2005).

18. R.K. Singh, H.R. Murty, S.K. Gupta, A.K. Dikshit, Integrated environment management in steel industries, Int. J. of Manag. Dec. Mak., 9 (2):103$128,(2008)$.

19. M. Segarra-Oña, A. Peiró-Signes, R. Verma, L., Miret-Pastor, Does Environmental Certification Help the Economic Performance of Hotels? Evidence from the Spanish Hotel Industry, Cornell Hosp. Q., 53(3):242256 (2012).

20. A. M. Turk, The benefits associated with ISO 14001 certification for construction firms: Turkish case, J. Clean. Prod., 17(5):559-569, (2009).

21. P. Barla, ISO 14001 certification and environmental performance in Quebec's pulp and paper industry, J. Envir. Eco. Manag., 53(3): 291-306, (2007).

22. M. Abbaspour, A. R. Karbassi y S. Khadivi, "Implementation of green management concepts in sport complexes", Int. J. Environ. Sci. Tech., 3(3): 213-219 (2006)

23. L.M., Sheppard, Environmental awareness. An overview of ISO 14000, Ceramic Industry, 149 (7): 20-21, (1999)

24. J A., Espí Rodriguez, Determinación de la sostenibilidad de la industria mineral de la cerámica y del vidrio mediante herramientas de gestión ambiental, Bol. Soc. Esp. Ceram. Vidr., 50(3): 151-160 (2011).

25. I. Heras-Saizarbitoria, J. F., Molina-Azorín, G.P.M. Dick, “ISO 14001 certification and financial performance: selection-effect versus treatment-effect", J. Clean. Prod., 19 (1):1-12, (2011a).

26. I. Heras-Saizarbitoria, G. Arana Landín, J. F. Molina-Azorín, “Do drivers matter for the benefits of ISO 14001?", Int. J. Oper. Prod. Man., 31(2):192 216, (2011b).

27. T. H. Arimura, N. Darnall, H. Katayama, Is ISO 14001 a gateway to more advanced voluntary action? The case of green supply chain management, J. Environ. Econ. Manag., 61 (2): 170-182, (2011).

28. S. Curkovic, R. Sroufe, Using ISO 14001 to promote a sustainable supply chain strategy, Business Strategy and the Environment, Bus. Strat. Env., 20 (2): 71-93 (2011).

29. D. Nawrocka, T. Brorson, T. Lindhqvist, ISO 14001 in environmental supply chain practices, J. Clean. Prod, 17(16): 1435-1443, (2009).

30. S. A. Melnyk, R.P. Sroufe, R. Calantone, Assessing the impact of environmental management systems on corporate and environmental performance, J. Oper. Manag., 21(3):329-351, (2003)

31. M. Russo, P. Börkey, E. Cubel, F. Lévêque F., F. Mas, Local sustainability and competitiveness: the case of the ceramic tile industry. Dublin: European Foundation for the Improvement of Living and Working Conditions. (1998)

32. ASCER, Información económica del sector español de baldosas cerámicas, Información económica. En http: / / www.ceramicadeespaña. es (2012).

33. S. Gabaldon, S. Lopez, J. B. Carda, J. B. Legislación y gestión medioambiental en la producción de baldosas cerámicas, Bol. Soc. Esp. Ceram. Vidr., 42 (3): 169-179, (2003).

34. Z. Shu, E., Monfort, J. Garcia-Ten, J. Zhou, J.L. Amoros, Y. Wang, A new cleaner process to prepare pressing-powder, Bol. Soc. Esp. Ceram. Vidr., 50 (5):235-244, (2011).

35. J. Alegre-Vidal, R. Lapiedra-Alcamí, R. Chiva-Gomez, Linking operations strategy and product innovatipon: an empirical study of Spanish ceramin tile producers, Res. Policy, 33:829-839, (2004).

36. J. Albors-Garrigós, J. L. Hervas-Oliver, La industria cerámica europea en el siglo XXI. Retos tecnológicos y desafíos de la próxima década, Bol. Soc. Esp. Ceram. Vidr. 45 (1):13-21, (2006).

37. E. Criado, Reflexiones sobre el futuro de la Industria Europea de la Cerámica, Bol. Soc. Esp. Ceram. Vidr., 46 (1): 39-44, (2007).

38. E. Tortajada-Esparza, D. Gabaldón-Estevan, I. Fernández-de-Lucio, I. La evolución tecnológica del distrito cerámico de Castellón: la contribución de la industria de fritas, colores y esmaltes. Bol. Soc. Esp. Ceram. Vidr., 47(2): 57-80 (2008)

39. J. Albors, J.L. Hervás, P. Márquez, Adopción contingente de tecnología de producción en el sector cerámico español. Un estudio empírico, Bol. Soc. Esp. Ceram. Vidr., 45(5): 338-345, (2006).

40. M. Segarra-Oña, A. Peiró-Signes, L. Miret-Pastor, J. Albors-Garrigós, ¿Eco-innovación, una evolución de la innovación? Análisis empírico en la industria cerámica española, Bol. Soc. Esp. Ceram. Vidr., 50 (5): 253-260, (2011).

41. S. Kuhtz, C., Zhou, V., Albino, DM., Yazan, Energy use in two Italian and Chinese tile manufacturers: A comparison using an enterprise inputoutput model, Energy, 35(1): 364-374, (2010).

42. D. Morrow, D. Rondinelli, Adopting Corporate Environmental Management Systems:: Motivations and Results of ISO 14001 and EMAS Certification, European Management Journal, 20(2):159-171, (2002).

43. A. Zutshi, A. Sohal, Environmental management system adoption by Australasian organisations: part 1: reasons, benefits and impediments, Technovation, 24(4):335-357, (2004). 
44. M.Segarra-Oña, L. Miret-Pastor, L., A. Peiró-Signes, Is The Food Industry Taking A Proactive Stance On Environmental Issues? Results From An Empirical Analysis. Review of Business Information Systems, 15:11-16, (2011)

45. J. Albors-Garrigós, P. Marquez-Rodríguez, J.L. Hervas-Oliver, Las relaciones fabricante distribuidor como elementos básicos de un modelo competitivo en el caso del cluster cerámico español. Análisis empírico de los factores moderadores, Bol. Soc. Esp. Ceram. Vidr., 47(6): 339-344, (2008).

46. J. Albors-Garrigós, P. Márquez-Rodríguez, M. Segarra-Oña, Internet como herramienta de creación de valor en sectores maduros. El caso de los productores y distribuidores cerámicos en España, Bol. Soc. Esp. Ceram. Vidr. 48(6):273-278, (2009).

47. K. B. Hendricks, V. R. Singhal, J. K. Stratman, The impact of enterprise systems on corporate performance: A study of ERP, SCM, and CRM system implementations, J. Oper. Manag., 25(1):65-82, (2007).

48. S. J Brown, J. B. Warner, Using daily stock returns: The case of event studies, J. Financ. Econ. 14: 205-258, (1985).

49. P. Asquith, P. Healy, K. Palepu, Earnings and stock splits, Account. Rev., 64(3): 387-403, (1989).

50. L.Y Dann, R. Masulis, D. Mayers, Repurchase tender offers and earnings information, J. Account. Econ., 14, (3), 217-251, (1991).

51. F. Degeorge, R. Zeckhauser, The reverse LB0 decision and firm performance: Theory and evidence, J. Financ., 48 (4):1323-1348, (1993).

52. D. J. Denis, D. K. Denis, Managerial discretion, organization structure, and corporate performance: A study of leveraged recapitalizations, J. Account. Econ., 16 (1-3): 209-236, (1993).

53. B. M. Barber, J. D. Lyon, Detecting abnormal operating performance: The empirical power and specification of test statistics, J. Finan. Econ., 41(3): 359-399, (1996).
54. G.E. Box, J. S. Hunter, W. G. Hunter, Estadística para Investigadores, Diseño, innovación y descubrimiento, $2^{a}$ Ed., Editorial Reverte, Barcelona (2008).

55. M. Segarra-Oña, B. de-Miguel-Molina, Concentración industrial sinérgica: Propuesta Metodológica y Aplicación a un Sector Industrial, Tec empresarial, 3 (1-2):65-72.

56. C. Carrascosa-López, M. Segarra-Oña, A. Peiró-Signes, B. SeguraGarcía-del-Río, Does It Pay to Be "Greener" than Legislation? An Empirical Study of Spanish Tile Industry, Journal of Sustainable Development, 5 (5):17-26 (2012).

57. M. Segarra-Oña, A. Peiró-Signes, B. de-Miguel-Molina, M. de-MiguelMolina, Análisis de la proactividad medioambiental en el sector industrial valenciano. ¿Qué buscan las empresas, mejorar la responsabilidad social corporativa o lavar su imagen?, Anals de la Real Academia de Cultura Valenciana, 87:227-258 (2012).

58. M. Segarra Oña, P. Merello Giménez, M. Segura Maroto, C. Maroto Álvarez, Peiró-Signes, A. Proactividad medioambiental en la empresa: clasificación empírica y determinación de aspectos clave, Tec Empresarial, 6 (1): 35-48, (2012).

59. J. L. Hervas-Oliver, J. Albors-Garrigos, The role of the firm's internal and relational capabilities in clusters: when distance and embeddedness are not enough to explain innovation, J. Econ. Geogr., 9 (2): 263-283, (2008).

60. F.X. Molina-Morales, The Territorial Agglomerations of Firms: A Socia Capital Perspective from the Spanish Tile Industry, Growth Change, 36 (1): 74-99.

Recibido: 29/02/2012

Aceptado: $17 / 12 / 2012$ 